\title{
Pareto efficiency in the jungle
}

\author{
Harold Houba ${ }^{1}$ - Roland Iwan Luttens ${ }^{2}$. \\ Hans-Peter Weikard ${ }^{3}$
}

Received: 8 December 2015 / Accepted: 31 March 2017 / Published online: 8 April 2017 (C) The Author(s) 2017. This article is an open access publication

\begin{abstract}
We revisit the jungle economy of Piccione and Rubinstein (Econ $\mathrm{J}$ 117(July):883-896, 2007) in which the allocation of resources is driven by coercion. In this economy the unique equilibrium satisfies lexicographic maximization. We show that relaxing the assumptions on consumption sets and preferences slightly, allocations other than lexicographic maximizers can be jungle equilibria. We attribute this result to single unilateral taking where a stronger agent takes goods from only one weaker agent. Once multiple unilateral takings are introduced, we show that jungle equilibria coincide with lexicographic maximization under weak assumptions. However, we also present examples of equilibria that are Pareto inefficient, where voluntary gift giving by stronger agents is needed to achieve Pareto efficiency and even voluntary trade has a role in the jungle.
\end{abstract}

$凶 \quad$ Roland Iwan Luttens

r.i.luttens@vu.nl

Harold Houba

harold.houba@vu.nl

http://staff.feweb.vu.nl/hhouba

Hans-Peter Weikard

hans-peter.weikard@wur.nl

http://www.socialsciences.wur.nl/enr/

1 Department of Econometrics, Tinbergen Institute, Vrije Universiteit Amsterdam, De Boelelaan 1105, 1081 HV Amsterdam, Netherlands

2 Department of Economics, Amsterdam University College, Vrije Universiteit Amsterdam, De Boelelaan 1105, 1081 HV Amsterdam, Netherlands

3 Wageningen School of Social Sciences, Wageningen University, Postbox 8130, 6700 EW Wageningen, Netherlands 
Keywords Power · Coercion · Jungle economy · Pareto efficiency · Gift giving · Trade

JEL Classification D51 · D61 · P52

\section{Introduction}

While the competitive market is based on voluntary exchange, the jungle economy is characterized by coercive exchange where stronger agents can take goods from weaker agents. The analysis of the jungle economy provides a complement to the Walrasian equilibrium model with which it shares existence and welfare properties (Piccione and Rubinstein 2007, P\&R hereafter). Embedded within the rich tradition of social contract theory following Hobbes and Locke, it facilitates a better understanding of the allocation of initial endowments, the exogenous primitive of the competitive equilibrium model. P\&R propose a stylized model in which coercion governs the exchange of resources in the jungle. Coercion is driven by the agents' preferences over bounded consumption sets and power relations that are described by an exogenous ranking of agents according to their strength. Weaker agents concede to stronger agents without engaging in costly conflict. The jungle economy mirrors the standard model of an exchange economy. The exogenous distribution of power in the jungle is the counterpart of the distribution of initial endowments in the market.

In a jungle equilibrium, a stronger agent no longer wants to take goods from any weaker agent nor from a pile of common goods, that no other agent holds. P\&R specify certain conditions on consumption sets and preferences under which a unique and Pareto efficient jungle equilibrium exists. ${ }^{1}$ This jungle equilibrium coincides with the unique lexicographic maximum in which all of the economy's resources are initially common goods and stronger agents take from the pile of common goods before weaker agents can take.

It is tempting to conclude from $\mathrm{P} \& \mathrm{R}$ 's intriguing analysis that exactly the particular strength relation assumed in their paper constitutes the main driving force behind the final distribution of resources in the jungle. However, this conclusion is somewhat premature. The goal of our paper is to provide a more nuanced view on the interaction of strength, preferences and holdings behind the jungle equilibrium concept. Intentionally, we do not deviate from P\&R's strength relation throughout the paper.

In our analysis we assume that initial holdings are distributed over the agents rather than being available as common goods, as is the case in P\&R. More generally, a stronger agent may take from any weaker agent or from the (remaining) pile of common goods. Under P\&R's assumptions on consumption sets and preferences, initial holdings are irrelevant for lexicographic maximization. The intuition is that each single taking improves the taker's welfare and thus only an allocation where the stronger agents are either satisfied or have acquired all goods can be an equilibrium. The initial distribution

\footnotetext{
1 These assumptions are compact and convex consumption sets and smooth, strongly monotone and strictly convex preferences.
} 
of resources among agents in jungle economies is relevant only to determine from whom a stronger agent takes.

However, once we relax the assumptions of strong monotonicity and strict convexity of preferences assumed by $\mathrm{P} \& \mathrm{R}$, the distribution of initial holdings matters. A single taking may not improve the taker's welfare such that an inefficient allocation can 'survive' as an equilibrium. Imagine an example of a jungle economy with a strong agent and two weaker agents, where the former holds Leontief preferences over pairs of shoes and currently holds no shoes. Suppose one weaker agent holds a left shoe and the other holds a right shoe. Since getting only a left shoe or only a right shoe does not increase the strong agent's utility, the strong agent will not take if restricted to a single taking. Therefore, the jungle is in equilibrium. ${ }^{2}$ In such a case the jungle equilibrium does not satisfy lexicographic maximization, nor is it Pareto efficient.

As this example illustrates, the jungle equilibrium concept fails to recognize that the stronger agent can gain by coercing both weaker agents even if each single taking does not improve the strong agent's welfare. We show that if we include multiple takings into the equilibrium concept, jungle equilibria coincide with lexicographic maximization under rather weak assumptions. ${ }^{3}$

Furthermore, with the use of an example, we derive a continuum of equilibria in which the strongest agent holds goods in excess of her satiation point. As the strongest agent has no incentive to dispose of excess goods, she may withhold them from weaker agents, who cannot take them. This withholding of goods is Pareto inefficient and only voluntary gift giving by stronger agents can remove this inefficiency. With another example, we show that even voluntary gift giving may sometimes be insufficient to achieve Pareto efficiency. Then, voluntary trade is needed.

These examples demonstrate why we believe that our analysis adds to a better understanding of the crucial assumptions underlying jungle economies. Pareto efficiency in the jungle is not a result of coercion alone. Interestingly, depending on the kind of preferences present in the jungle, voluntary gift giving and voluntary trade, behavior that is in sharp contrast to coercion, is needed to keep the jungle efficient. Thus, our conclusions diverge strikingly from $P \& R$, who see no role for gift giving and trade in the jungle.

We proceed as follows. Section 2 presents a formal account of a jungle economy with initial holdings of which P\&R's jungle economy is a special case. Section 3 investigates lexicographic maximization and provides two examples to motivate our analysis. The jungle equilibrium with multiple unilateral takings is investigated in Sect. 4. The subtle role of withholding, voluntary gift giving and voluntary trade is discussed in Sect. 5. Section 6 concludes.

\footnotetext{
2 For the sake of the argument, assume the weaker agents cannot take from each other.

3 These assumptions are non-empty, compact and strictly comprehensive consumption sets and complete, transitive and continuous preferences.
} 


\section{Preliminaries}

We consider a finite set of agents $N=\{1, \ldots, n\}$ of size $n \geq 2$ and a finite number $m \geq 1$ of goods that are present in positive quantities. We refer to $z^{i}$ as the holdings and to $x^{i}$ as the consumption of agent $i \in N$. Consumption is feasible when $x^{i} \leqq z^{i}$ for all $i \in N .^{4}$ The consumption set of agent $i$ is denoted $C^{i} \subset \mathbb{R}_{+}^{m}$. This set is nonempty, compact and strictly comprehensive, i.e., for all $z^{i} \in C^{i}$ and $\hat{z}^{i} \in \mathbb{R}_{+}^{m}$ such that $\hat{z}^{i} \leq z^{i}$ it holds that $\hat{z}^{i}$ lies in the interior of $C^{i}$. As in P\&R, an agent's holdings (and consumption) cannot exceed her consumption set. The preference relation of agent $i$ on $C^{i}$, denoted $\succeq^{i}$, is complete, transitive and continuous. Its symmetric and asymmetric parts are denoted $\sim^{i}$ and $\succ^{i}$ respectively.

An allocation $z=\left(z^{1}, \ldots, z^{n}, z^{n+1}\right)$ assigns holdings to each agent in $N$, while $z^{n+1} \in \mathbb{R}_{+}^{m}$ indicates the bundle of common goods that is held by none of the agents. The economy's total endowment equals $\widetilde{\omega} \in \mathbb{R}_{+}^{m}$. Unlike P\&R we allow that initial holdings are allocated to the agents. Initial holdings are defined as the feasible allocation $\omega=\left(\omega^{1}, \ldots, \omega^{n}, \omega^{n+1}\right)$. An allocation $z$ is feasible when $\sum_{i=1}^{n+1} z^{i}=\widetilde{\omega}$ and $z^{i} \in C^{i}$ for all $i \in N .^{5}$ A feasible allocation $z$ is Pareto efficient if there does not exist another feasible allocation $\hat{z}$ such that $\hat{z} \succeq^{i} z$ for all $i \in N$ and $\hat{z} \succ^{i} z$ for at least one $i \in N$.

Coercion governs the exchange of goods in the jungle economy and it is driven by the agents' preferences and strengths. The order of the agents reflects their strength. As in the jungle economy of $P \& R$, the strength relation is extreme. The strongest agent of any pair of agents has the power to take everything that the weaker agent possesses, while the weaker agent cannot take anything from the stronger agent. ${ }^{6}$ The agents in $N$ are ordered such that agent 1 is stronger than agent 2 , who is stronger than agent 3 , and so on. Thus $i<j$ implies that $i$ is stronger than $j$.

Thus far, we have defined an economy driven by coercion as a tuple $\left\langle N,\left\{C^{i}, \succeq^{i}\right\}_{i \in N}\right.$, $\omega\rangle$. This tuple extends the jungle economy of P\&R by introducing the initial holdings $\omega$. Furthermore, in the jungle economy of $\mathrm{P} \& \mathrm{R}$ each $C^{i}$ is a convex set and preferences in the jungle economy are strongly monotone and strictly convex. ${ }^{7}$ We relax this assumption later on.

An agent's feasible consumption is the set of bundles that this agent is able to reach from her own and the weaker agents' current holdings. It is convenient to define $y^{i, j} \leqq z^{j}$ as agent $i$ 's unilateral takings that result from a single coercive exchange between agents $i$ and $j>i$.

\footnotetext{
4 Vector inequalities: we write $a \leqq b$ if for all vector components $k$ we have $a_{k} \leq b_{k} ; a \leq b$ if for all vector components $k$ we have $a_{k} \leq b_{k}$ and there exist at least one component for which the inequality is strict; and $a<b$ if for all vector components $k$ we have $a_{k}<b_{k}$. Furthermore, $\subseteq$ denotes a subset and $\subset$ a strict subset.

5 We include $z^{i} \in C^{i}$ into the definition of feasible allocations because technically speaking agent $i$ 's preferences on $\mathbb{R}_{+}^{m} \backslash C^{i}$ are undefined.

6 The interpretation is that the weaker agent concedes to the stronger agent and does not initiate a costly conflict knowing it will be lost for sure.

7 In fact, monotone and strictly convex preferences imply strongly monotone preferences. However, for the sake of consistency with P\&R, we keep referring to the preferences in the jungle as strongly monotone and strictly convex.
} 
As a benchmark, we adopt the notion of jungle equilibrium of $\mathrm{P} \& \mathrm{R}$ in which stability against unilateral takings by stronger agents is the key idea.

Definition 1 A jungle equilibrium is a feasible allocation $z$ for which there does not exist an ordered pair of agents $i, j$, unilateral takings $y^{i, j}$ and a feasible bundle $\hat{z}^{i}=z^{i}+y^{i, j} \in C^{i}$ such that $\hat{z}^{i} \succ^{i} z^{i}$.

Note that the definition of a jungle equilibrium does not refer to the initial allocation which may assign goods to the agents or, as in $\mathrm{P} \& \mathrm{R}$, to the pile of common goods.

In what follows, besides coercion, we will also consider gift giving and trade in the jungle (cfr. Sect. 5). We define unilateral gift giving of good $k$ from agent $i$ to agent $j>i$ as a change from feasible allocation $z$ to feasible allocation $\hat{z}$ such that $\hat{z}_{k}^{i}<z_{k}^{i}$ and $\hat{z}_{k}^{j}>z_{k}^{j}$. We define bilateral trade between agents $i$ and $j$ of goods $k$ and $l$ as a change from feasible allocation $z$ to feasible allocation $\hat{z}$ such that $\hat{z}_{k}^{i}<z_{k}^{i}$ and $\hat{z}_{k}^{j}>z_{k}^{j}$ while $\hat{z}_{l}^{i}>z_{l}^{i}$ and $\hat{z}_{l}^{j}<z_{l}^{j} .8$

\section{Lexicographic maximization}

In the jungle economy of $\mathrm{P} \& \mathrm{R}$, where the initial allocation is just a pile of common goods, a jungle equilibrium can be obtained when agents take in the order of strength (P\&R, Proposition 1). We refer to the result of such a procedure as a lexicographic maximizer, which we define as follows:

Definition 2 A lexicographic maximizer is a feasible allocation $\bar{z}$ such that for every agent $i \in N$ it holds that $\bar{z}^{i} \succeq^{i} z^{i}$ for all $z^{i} \in\left\{x \in C^{i} \mid x \leqq \tilde{\omega}-\sum_{j=1}^{i-1} \bar{z}^{j}\right\}$.

It is useful to denote the set of lexicographic maximizers by $\bar{Z}$, i.e. the set of allocations $\bar{z}$ that satisfy Definition 2 . Assuming strongly monotone and strictly convex preferences, the jungle economy has a unique lexicographic maximum and hence a unique jungle equilibrium ( $\mathrm{P} \& \mathrm{R}$, Proposition 3 ).

However, when the assumption of strongly monotone and strictly convex preferences of $\mathrm{P} \& \mathrm{R}$ is relaxed to monotone and convex preferences, to allow for Leontief preferences, there may no longer be a unique lexicographic maximizer as Example 1 illustrates.

Example 1 Consider an economy with two agents and two goods. The economy's total resources are $\widetilde{\omega}=(2,1)$. The agents' consumption sets are identical and given by $C^{1}=C^{2}=\left\{x \in \mathbb{R}_{+}^{2} \mid x \leqq \widetilde{\omega}\right\}$ for simplicity. For $i=1,2$, agent $i$ 's best element of $\succeq^{i}$ on $C^{i}$ maximizes the Leontief preferences $\min \left\{z_{1}^{i}, z_{2}^{i}\right\}$. It is easy to see that allocations

$$
\begin{aligned}
\omega^{1} & =(1,1), \omega^{2}=(1,0) \text { and common goods } \omega^{3}=(0,0) \text { and } \\
\omega^{\prime 1} & =(1,1), \omega^{\prime 2}=(0,0) \text { and common goods } \omega^{\prime 3}=(1,0)
\end{aligned}
$$

\footnotetext{
${ }^{8}$ For the purpose of this paper it is sufficient to consider trade of two goods only. We therefore abstain from giving a more general definition of trade.
} 
both satisfy Definition 2 and are both jungle equilibria according to Definition 1 because neither agent 1 nor agent 2 can gain from unilateral takings.

An agent with Leontief preferences may not have an incentive to take a good, because a larger bundle, even though it belongs to her consumption set, is not necessarily better.

Moreover, there may exist jungle equilibria that are not lexicographic maximizers. Example 2 illustrates this argument.

Example 2 Consider an economy with two agents and three goods. The economy's total resources are $\widetilde{\omega}=(1,1,1)$. The agents' consumption sets are identical and given by $C^{1}=C^{2}=\left\{x \in \mathbb{R}_{+}^{3} \mid x \leqq \widetilde{\omega}\right\}$ for simplicity. For $i=1,2$, agent $i$ 's best element of $\succeq^{i}$ on $C^{i}$ maximizes the Leontief preferences $\min \left\{z_{1}^{i}, z_{2}^{i}, z_{3}^{i}\right\}$. For all allocations $\omega$, the unique lexicographic maximizer $\bar{z}$ is given by $\bar{z}^{1}=\widetilde{\omega}$ and $\bar{z}^{2}=\bar{z}^{3}=0$. However, for $\alpha \in[0,1]$,

$$
\omega^{1}=(1, \alpha, \alpha), \omega^{2}=(0,1-\alpha, 0) \text { and common goods } \omega^{3}=(0,0,1-\alpha)
$$

form an equilibrium according to Definition 1 because neither agent 1 nor agent 2 can gain from unilateral takings. For $\alpha<1$, it differs from the lexicographic maximum and it is Pareto inefficient because $z^{1}=(\alpha, \alpha, \alpha), z^{2}=(1-\alpha, 1-\alpha, 1-\alpha)$ and $z^{3}=(0,0,0)$ is welfare improving. Besides permutations, no other equilibria exist.

There are two intriguing issues about this example. First, the jungle equilibrium concept fails to recognize that stronger agents might evaluate the aggregate of multiple unilateral takings instead of a single unilateral taking. In the next section, we introduce multiple unilateral takings in the jungle. Second, for initial holdings $\omega^{1}=(1, \alpha, \alpha)$, the amount $1-\alpha$ of good 1 does not contribute additional welfare to agent 1 and this agent is indifferent between keeping it or disposing it. This observation gives a glimpse of the issue of withholding, which we discuss in Sect. 5.

Finally, note that unused resources can also occur in an equilibrium of an exchange economy. As Randon and Simmons (2017) explain for a famous example in Scarf (1960), such cases may result from unequally distributed initial holdings and rigid (Leontief) preferences.

\section{The jungle economy with multiple unilateral takings}

In this section, we include multiple unilateral takings into the jungle equilibrium concept and show that all jungle equilibria with multiple unilateral takings are lexicographic maximizers.

Formally, an agent $i$ does not only consider a single unilateral taking from a weaker agent $j$, as in a jungle equilibrium, but rather considers multiple unilateral takings from some or all weaker agents and from the pile of common goods. We denote agent $i$ 's multiple unilateral takings by the tuple $y^{i}=\left(y^{i, i+1}, \ldots, y^{i, n+1}\right)$. Given allocation $z$, agent $i$ 's multiple unilateral takings $y^{i}$ are feasible if 


$$
z^{i}+\sum_{j=i+1}^{n+1} y^{i, j} \in C^{i} \text { and } y^{i, j} \leqq z^{j} \text { for all } j \in\{i+1, \ldots, n+1\} .
$$

We define the jungle equilibrium with multiple unilateral takings as follows:

Definition 3 A jungle equilibrium with multiple unilateral takings is a feasible allocation $z$ such that there does not exist an agent $i \in N$ and feasible multiple unilateral takings $y^{i}$ for which $z^{i}+\sum_{j=i+1}^{n+1} y^{i, j} \succ^{i} z^{i}$.

Then, the following equivalence holds:

Theorem 1 Each lexicographic maximizer $\bar{z} \in \bar{Z}$ is a jungle equilibrium with multiple unilateral takings and vice versa.

Proof The result follows from the following equivalences: $\bar{z} \in \bar{Z} \Leftrightarrow$ for each $i \in N$ and feasible multiple unilateral takings $y^{i}$ it holds that $\bar{z}^{i} \succeq^{i} \bar{z}^{i}+\sum_{j=i+1}^{n+1} y^{i, j} \Leftrightarrow$ for each $i \in N$ there does not exist feasible multiple unilateral takings $y^{i}$ such that $\bar{z}^{i}+\sum_{j=i+1}^{n+1} y^{i, j} \succ^{i} \bar{z}^{i} \Leftrightarrow \bar{z}$ is a jungle equilibrium with multiple unilateral takings.

Note that $\bar{Z}$ is non-empty and therefore a jungle equilibrium with multiple unilateral takings always exists. Recall that consumption sets are non-empty, compact and strictly comprehensive and preferences are complete, transitive and continuous. Therefore, the assumptions for Theorem 1 are rather weak.

\section{Unilateral gift giving and bilateral trade}

In principle, an agent is not forced to consume all her holdings and may freely dispose or waste some of the resources available to her. In the jungle economy of P\&R agents may freely dispose goods. This is captured by the assumption that the consumption set is comprehensive. But given strongly monotone preferences in the jungle economy of P\&R, all agents consume their holdings in equilibrium. Example 2, however, illustrates that the distinction between holdings and consumption is more subtle and can matter. Holdings that are not consumed are withheld from other agents in the economy. We now investigate withholding of goods in equilibria with multiple unilateral takings by means of two additional examples.

The first of these is similar in spirit to Example 2 but differs in that a continuum of jungle equilibria with multiple takings are Pareto inefficient due to withholding, a phenomenon that is not present in Example 2.

Example 3 Consider an economy with two agents and two goods. The economy's total resources are $\widetilde{\omega}=(2,1)$. The agents' consumption sets are identical and given by $C^{1}=C^{2}=X=\left\{x \in \mathbb{R}_{+}^{2} \mid x \leqq \widetilde{\omega}\right\}$ for simplicity. Agent 1's best element of $\succeq^{1}$ on $C^{1}$ maximizes the Leontief preferences $\min \left\{z_{1}^{1}, z_{2}^{1}\right\}$ and agent 2's best element of $\succeq^{2}$ on $C^{2}$ maximizes $\sqrt{z_{1}^{2}}+\sqrt{z_{2}^{2}}$. For all allocations $\omega$, the set of lexicographic maximizers $\bar{Z}$ is given by 


$$
\begin{aligned}
& \left\{\left(\bar{z}^{1}, \bar{z}^{2}, \bar{z}^{3}\right) \in C^{1} \times C^{2} \times X \mid \bar{z}^{1}=(1+\varepsilon, 1),\right. \\
& \left.\bar{z}^{2}=(1-\varepsilon, 0), \bar{z}^{3}=(0,0), \varepsilon \in[0,1]\right\} .
\end{aligned}
$$

This set coincides with the set of jungle equilibria with multiple unilateral takings. For $\varepsilon>0$, the lexicographic maximum is Pareto inefficient because $z^{1}=(1,1), z^{2}=$ $(1,0)$ and $z^{3}=(0,0)$ is welfare improving. So, only the lexicographic maximizer corresponding to $\varepsilon=0$ is Pareto efficient. Withholding can be said to occur whenever $\varepsilon>0$.

The economic issue, left open in this paper, is whether agent 1 has an incentive to keep or voluntarily give away her excess holdings $\varepsilon>0$ of good 1 . By keeping $\varepsilon$ of good 1, this agent withholds it from agent 2, for whom it would increase welfare. Voluntary gift giving is needed to restore Pareto efficiency in this example.

From Example 3 it seems that only voluntary gift giving by stronger agents can remedy Pareto inefficiency. However, in our final example we contend that this is not necessarily true. We argue that even voluntary bilateral trade can have a role in the jungle.

Example 4 Consider an economy with two agents and two goods. The economy's total resources are $\widetilde{\omega}=(1,1)$. Agent 1 's consumption set is given by $C^{1}=$ $\left\{x \in \mathbb{R}_{+}^{2} \mid x_{1}+x_{2} \leq 1\right\}$ and, for simplicity, agent 2's consumption set is given by $C^{2}=\left\{x \in \mathbb{R}_{+}^{2} \mid x \leqq \widetilde{\omega}\right\}$ and $X=\left\{x \in \mathbb{R}_{+}^{2} \mid x \leqq \widetilde{\omega}\right\}$. Agent 1 's best element of $\succeq^{1}$ on $C^{1}$ maximizes the preferences $z_{1}^{1}+z_{2}^{1}$ and agent 2's best element of $\succeq^{2}$ on $C^{2}$ maximizes $\sqrt{z_{1}^{2}}+\sqrt{z_{2}^{2}}$. For all allocations $\omega$, the set of lexicographic maximizers $\bar{Z}$ is given by

$$
\begin{aligned}
& \left\{\left(\bar{z}^{1}, \bar{z}^{2}, \bar{z}^{3}\right) \in C^{1} \times C^{2} \times X \mid \bar{z}^{1}=(\varepsilon, 1-\varepsilon),\right. \\
& \left.\bar{z}^{2}=(1-\varepsilon, \varepsilon), \bar{z}^{3}=(0,0), \varepsilon \in[0,1]\right\} .
\end{aligned}
$$

This set coincides with the set of jungle equilibria with multiple unilateral takings (Theorem 1). Obviously, no withholding occurs. For $\varepsilon \neq \frac{1}{2}$, however, the lexicographic maximizer is Pareto inefficient because $z^{1}=\left(\frac{1}{2}, \frac{1}{2}\right), z^{2}=\left(\frac{1}{2}, \frac{1}{2}\right)$ and $z^{3}=(0,0)$ is welfare improving. So, only the lexicographic maximizer corresponding to $\varepsilon=\frac{1}{2}$ is Pareto efficient. It can only be reached from allocations with $\varepsilon \neq \frac{1}{2}$ by voluntary trade in which goods 1 and 2 are exchanged one-for-one.

As before, the economic issue remains whether agent 1 has an incentive to voluntarily trade with agent 2 or not.

\section{Conclusion}

This paper provides a detailed analysis of the jungle economy as first described by P\&R. Our work can be interpreted as a sensitivity analysis regarding some crucial 
assumptions introduced by $\mathrm{P} \& \mathrm{R}$. While throughout the paper we maintain the assumption that stronger agents have coercive power over weaker agents, we introduce two extensions of P\&R's benchmark model of a jungle economy. First, we allow arbitrary initial holdings of the agents. Second, we allow agents in the jungle to have Leontief preferences. In this case, Example 2 shows that stronger agents may not be able to gain from a single unilateral taking even if there are feasible allocations that make the stronger agents better off. Hence, jungle equilibria are not necessarily lexicographic maximizers. We respond to this finding by introducing the concept of a jungle economy with multiple unilateral takings and show that lexicographic maximization implements jungle equilibria with multiple unilateral takings under weak assumptions. However, Example 3 shows that a jungle equilibrium with multiple unilateral takings need not be Pareto efficient. Stronger agents can withhold goods they do not wish to consume from weaker agents. Only voluntary gift giving can restore Pareto efficiency in this case. Example 4 shows that, in some non-generic cases, even voluntary bilateral trade is necessary to achieve Pareto efficiency.

The microeconomic idea of an efficient jungle has its philosophical underpinning in John Locke's (1690, section 31) no-spoilage proviso. In his famous "Second Treatise of Government" Locke argues that legitimate property rights are incompatible with wasting resources. Locke's second proviso that one can only privately acquire goods from the common pool as long as "there is enough, and as good left in common for others" (section 27) is, however, violated in the jungle.

Open Access This article is distributed under the terms of the Creative Commons Attribution 4.0 International License (http://creativecommons.org/licenses/by/4.0/), which permits unrestricted use, distribution, and reproduction in any medium, provided you give appropriate credit to the original author(s) and the source, provide a link to the Creative Commons license, and indicate if changes were made.

\section{References}

Locke J (1690) Two treatises of government. In: Laslett P (ed) 2nd edn. Cambridge University Press, Cambridge (reprinted by Peter Laslett in 1967)

Piccione M, Rubinstein A (2007) Equilibrium in the jungle. Econ J 117(July):883-896

Randon E, Simmons P (2017) A top dog tale with preference Journal Econ/Zeitschrift fur Nationalokonomie 120(1):47-63

Scarf H (1960) Some examples of global instability of the competitive equilibrium. Int Econ Rev 1(3):157172 